\title{
Inhibitory Effect of Hydroxyindoles and their Analogues on Human Melanoma Tyrosinase
}

\author{
Yoshimitsu Yamazaki* and Yasuhiro Kawano \\ Institute for Biological Resources and Functions, National Institute of Advanced Industrial \\ Science and Technology, 1-1-1 Higashi, Tsukuba, Ibaraki 305-8566, Japan. \\ Fax: +8 12986160 63. E-mail: y.yamazaki@aist.go.jp \\ * Author for correspondence and reprint requests \\ Z. Naturforsch. 65 c, 49-54 (2010); received October 22, 2009
}

A recent study showed that $N$-acylserotonin derivatives have strong inhibitory activity against tyrosinase. To clarify the role of the 5-hydroxy group in the indole ring, 2-, 4-, 5-, 6-, and 7-hydroxyindole and 11 related compounds such as 5-hydroxyindan and 6-hydroxyquinoline were tested for their inhibition of catecholase activity of tyrosinase from human HMVII melanoma cells. 6-Hydroxyindole (5) and 7-hydroxyindole (6) were potent inhibitors, while 5 -hydroxyindole (4) was a weaker inhibitor than the above-mentioned compounds $\left(\mathrm{IC}_{50}=\right.$ $20,79,366$, and $342 \mu \mathrm{M}$ for $\mathbf{5 , 6 , 4}$, and kojic acid, respectively). 2-Hydroxycarbazole was also active $\left(\mathrm{IC}_{50}=190 \mu \mathrm{M}\right)$, 5-hydroxyindan, 4-aminophenol, and harmalol were slightly active, and other compounds were inactive as an inhibitor. A similar pattern of inhibition was found with these compounds against mouse B16 melanoma tyrosinase, but with some differences from that for HMV-II tyrosinase. Kinetic analysis with HMV-II tyrosinase showed that the inhibition by hydroxyindoles $\mathbf{4}, \mathbf{5}$, and $\mathbf{6}$ was competitive with respect to the substrate LDOPA. Melanin formation in HMV-II cells was suppressed by $14 \%$ with $10 \mu \mathrm{m} \mathbf{5}$ without cytotoxicity, but 30 or $100 \mu \mathrm{m} \mathbf{5}$ decreased the cell viability. The present results suggest that 6-hydroxyindole is a potential and useful pharmacophore of antimelanogenic agents and that the position of a phenolic hydroxy group in a specific heterocyclic ring such as in indole is possibly optimized to yield more active inhibitors for tyrosinase.

Key words: Tyrosinase, Inhibitor, Hydroxyindole

\section{Introduction}

Tyrosinase (EC 1.14.18.1) inhibitors are widely studied for developing efficient skin-lightening agents (Chang, 2009; Khan, 2007). Recently, we found that $N$-acylserotonin derivatives with phenolic substituents, such as $N$-caffeoylserotonin, strongly inhibited tyrosinase from mouse and human melanoma cells (Yamazaki et al., 2009). The inhibitory activity of these compounds is closely connected with the presence of the 5-hydroxy group in the indole ring. Almost a half century ago, 5-hydroxyindole (4) was reported as a tyrosinase inhibitor (Seiji, 1961), and later its activity was compared with those of some tryptophan derivatives and metal chelators (Pomerantz, 1963), but no detailed structure-activity relationship (SAR) study concerning the 5-hydroxyindole moiety has been reported. Phenolic compounds are the main class of tyrosinase inhibitors as alternative substrates. While many studies were carried out on the effect of the number and position of the hydroxy groups in stilbene (Kim et al., 2002), chal- cone (Nishida et al., 2007), flavone (Kubo et al., 2000), and phenylalkylamide skeletons (Okombi et al., 2006; Cho et al., 2006) on tyrosinase inhibitory activity, such an analysis has been rarely reported for $N$-heterocyclic phenols. On the other hand, previous screening of tyrosinase inhibitors has been done mostly with mushroom tyrosinase (Cho et al., 2006; Tamura et al., 2007). Some studies used tyrosinase from mouse B16 melanoma cells (Kim et al., 2002), and fewer studies used human tyrosinase (Okombi et al., 2006). Mushroom tyrosinase showed, with some compounds, a considerably different behaviour from those of the mammalian enzymes, and the B16 tyrosinase also does not necessarily behave in parallel with a human melanoma tyrosinase (Yamazaki et al., 2009). Screening with tyrosinase from human melanocytes is beneficial in developing skin lighteners, but because of some difficulty in cultivating normal melanocytes the next best way would be the use of human melanoma tyrosinase. The nucleotide and amino acid sequences of a human 
melanoma tyrosinase were reported to be nearly identical to those of the human melanocyte tyrosinase (Bouchard et al., 1989), suggesting that their enzymatic properties are, if not the same, very similar to each other.

Here, we report several hydroxyindoles as potent inhibitors of HMV-II human melanoma tyrosinase in L-3,4-dihydroxyphenylalanine (L-DOPA) oxidation, with SAR focused on the position of the hydroxy group in indole and several related ring structures.

\section{Material and Methods}

\section{Reagents}

Indole (1), 2- (2), 4- (3), and 6-hydroxyindole (5), harmalol (14), and harmaline (15) were purchased from Wako Pure Chemicals Inc. (Osaka, Japan), 5-hydroxyindole (4), 5-hydroxyindan (7), 3- (11) and 4-aminophenol (10), 4-hydroxycarbazole (12), 3,4-dihydro-7-hydroxy-2(1H)-quinolinone (16), and kojic acid (17) were from Tokyo Chemical Industries Ltd. (Tokyo, Japan), and 7-hydroxyindole (6) from Kanto Chemical Company Inc. (Tokyo, Japan). 2-Hydroxycarbazole (13), 6(8) and 8-hydroxyquinoline (9), and mushroom tyrosinase were obtained from Sigma-Aldrich Co. (St. Louis, MO, USA).

\section{Tyrosinase assay}

Human HMV-II melanoma cells (Dai-NipponSumitomo Pharmaceuticals Inc., Osaka, Japan) were grown in RPMI-1640 medium containing $15 \%$ fetal bovine serum (FBS). Tyrosinase was extracted from the cells according to the published method (Yamazaki et al., 2009). Assay solutions were prepared by mixing $0.94 \mathrm{ml} 50 \mathrm{~mm}$ phosphate buffer ( $\mathrm{pH}$ 6.8) containing $2.6 \mathrm{~mm} \mathrm{L-DOPA}$ and $10 \mu \mathrm{l}$ ethanol containing $0-30 \mathrm{~mm}$ test compound, unless otherwise stated. The reaction was started by adding the enzyme solution $(50 \mu \mathrm{l})$ to the substrate solutions prewarmed to $37^{\circ} \mathrm{C}$, and the dopachrome formation during the incubation for $5 \mathrm{~min}$ was determined by light absorption at $475 \mathrm{~nm}\left[\varepsilon=3700 \mathrm{~m}^{-1} \mathrm{~cm}^{-1}\right.$ (Kubo et al., 2003)]. Usually, 2.5-3 mU enzyme (Cabanes et al., 1984) were applied per assay. Experiments with tyrosinase extracted from B16 mouse melanoma cells (Riken Cell Bank, Tsukuba, Japan) and mushroom tyrosinase were done in the same way as described above. $\mathrm{IC}_{50}$ values were obtained from
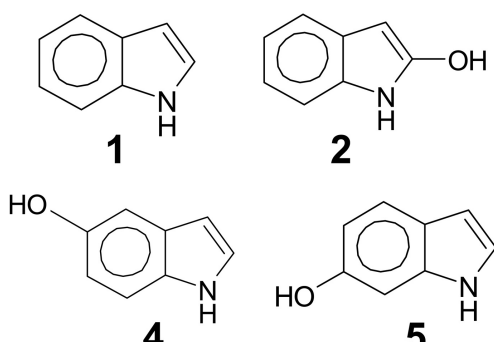
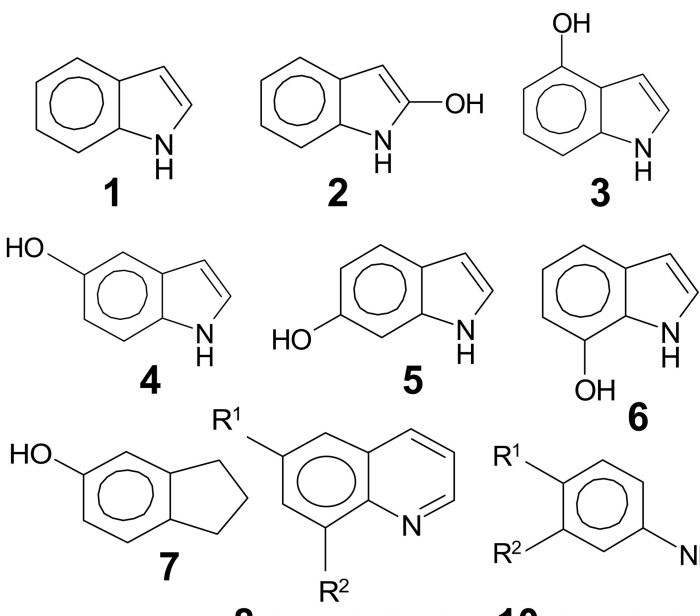

6

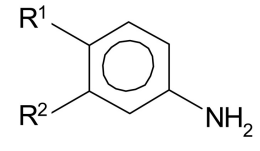

8: $R^{1}=\mathrm{OH}, \mathrm{R}^{2}=\mathrm{H}$

10: $\mathrm{R}^{1}=\mathrm{OH}, \mathrm{R}^{2}=\mathrm{H}$

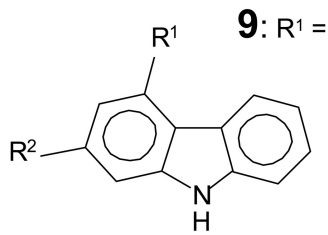

12: $R^{1}=O H, R^{2}=H$

13: $R^{1}=H, R^{2}=O H$

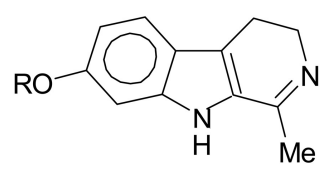

14: $\mathrm{R}=\mathrm{H}$

15: $R=M e$<smiles>O=C1CCc2ccc(O)cc2N1</smiles>

Fig. 1. Chemical structures of the compounds.

plots of concentration $v$ s. relative activity (\% of control).

\section{Antimelanogenic assay}

HMV-II cells were cultured in 96-well plates $\left(1 \cdot 10^{4}\right.$ cells in $0.2 \mathrm{ml}$ medium per well) for $1 \mathrm{~d}$. The medium of each well was changed with fresh medium $(0.2 \mathrm{ml})$ containing $500 \mu \mathrm{M}$ theophylline, and then $2 \mu \mathrm{l}$ ethanol solutions containing $0-10 \mathrm{~mm}$ test compounds were added to the wells. After incubation for $3 \mathrm{~d}$, the medium was aspirated off, and cell viability was assayed with a tetrazolium reagent (CellTiter $96^{\circledR}$, Promega Corp., Madison, WI, USA). The intracellular melanin content was measured by the published method (Liu et al., 2007), but with some modifications. Cells were cultured in 12 -well plates $\left(2 \cdot 10^{5}\right.$ cells in $2 \mathrm{ml}$ medium per well) for $1 \mathrm{~d}$, and $40 \mathrm{~mm}$ 
theophylline solution in water $(25 \mu \mathrm{l}$ each $)$ and ethanol solutions of test compounds ( $2 \mu \mathrm{l}$ each) were added to the wells. After $3 \mathrm{~d}$ of incubation, cells of each well were collected by a cell scraper, washed with phosphate-buffered saline, and then homogenized in $0.2 \mathrm{ml} 1 \mathrm{M} \mathrm{NaOH}$ by sonication. The absorbance at $415 \mathrm{~nm}$ of the homogenates was measured to evaluate the intracellular melanin content.

\section{Results and Discussion}

Among the tested compounds (Fig. 1), 5- (4), 6- (5), and 7-hydroxyindole (6), and 2-hydroxycarbazole (13) clearly inhibited the L-DOPA oxidation by HMV-II melanoma tyrosinase. The typical result for the dose-dependency of inhibition is shown in Fig. 2, and $\mathrm{IC}_{50}$ values and percentage of inhibition at a concentration of $100 \mu \mathrm{M}$ are summarized in Table I. The order of the activity strength was $5>\mathbf{6}>\mathbf{1 3}>$ kojic acid (17, the usual antimelanogenic agent) $\geq \mathbf{4}$, as shown by the $\mathrm{IC}_{50}$ values. 5-Hydroxyindan (7), 4-aminophenol (10), and harmalol (14) indicated marginal inhibitory activities, while 2- (2) and 4-hydroxyindole (3), 6- (8) and 8-hydroxyquinoline (9), 3-aminophenol (11), 4-hydroxycarbazole (12), harmaline (15), 3,4-dihydro-7-hydroxy-2(1H)quinolinone (16), and indole (1) were inactive in inhibiting this tyrosinase. A similar pattern of inhibitory activity with these compounds was found with B16 mouse melanoma tyrosinase, but 4-hydroxyindole (3) weakly inhibited B16 tyrosinase in contrast to the inactivity against HMV-II enzyme (Table I). Importantly, 6-hydroxyindole (5) and 7-hydroxyindole (6) were stronger inhibitors than 5-hydroxyindole (4) and kojic acid (17) against B16 tyrosinase as well as HMV-II tyrosinase. In addition, 4- (3), 5- (4) and 6-hydroxyindole (5), and kojic acid (17) inhibited mushroom tyrosinase $\left[\mathrm{IC}_{50}=(31 \pm 7),(77 \pm 4),(51 \pm 8)\right.$, and $(59 \pm 6) \mu \mathrm{M}$ (mean $\pm \mathrm{sd}, n=3$ ), respectively, under the same assay conditions as above], while 2-hydroxyindole (2), 7-hydroxyindole (6), and 6-hydroxyquinoline $(8)$ promoted the oxidation of L-DOPA by the mushroom enzyme [initial rates $=(144 \pm 7),(136 \pm 9)$, and $(176 \pm 12) \%$ of the control (mean $\pm \mathrm{sd}, n=3$ ) in the presence

Table I. Tyrosinase inhibitory activity of hydroxyindoles and the related compounds ${ }^{\mathrm{a}}$.

\begin{tabular}{|c|c|c|c|c|}
\hline \multirow[t]{3}{*}{ Compound } & \multicolumn{4}{|c|}{ Cell line } \\
\hline & \multicolumn{2}{|c|}{ HMV-II } & \multicolumn{2}{|c|}{ B16 } \\
\hline & $\mathrm{IC}_{50}[\mu \mathrm{M}]$ & $\begin{array}{l}\text { Inhibition at } \\
100 \mu \mathrm{M}^{\mathrm{b}}(\%)\end{array}$ & $\mathrm{IC}_{50}[\mu \mathrm{M}]$ & $\begin{array}{l}\text { Inhibition at } \\
100 \mu \mathrm{M}^{\mathrm{b}}(\%)\end{array}$ \\
\hline Indole (1) & - & $-13 \pm 8$ & - & $-8 \pm 3$ \\
\hline 2-Hydroxyindole (2) & - & $-7 \pm 6$ & - & $1 \pm 5$ \\
\hline 4-Hydroxyindole (3) & - & $1 \pm 5$ & $1055 \pm 69$ & $9 \pm 4$ \\
\hline 5-Hydroxyindole (4) & $366 \pm 41$ & $27 \pm 3$ & $45 \pm 9$ & $75 \pm 1$ \\
\hline 6-Hydroxyindole (5) & $20 \pm 3$ & $83 \pm 4$ & $18 \pm 10$ & $89 \pm 9$ \\
\hline 7-Hydroxyindole (6) & $79 \pm 13$ & $54 \pm 2$ & $19 \pm 3$ & $81 \pm 3$ \\
\hline 5-Hydroxyindan (7) & $>1000$ & $9 \pm 4$ & $>1000$ & $7 \pm 2$ \\
\hline 6-Hydroxyquinoline (8) & - & $-1 \pm 4$ & - & $-5 \pm 4$ \\
\hline 8-Hydroxyquinoline (9) & - & $1 \pm 5$ & - & $0 \pm 2$ \\
\hline 4-Aminophenol (10) & - & $13 \pm 3$ & $173 \pm 59$ & $40 \pm 6$ \\
\hline 3-Aminophenol (11) & - & $-4 \pm 4$ & - & $-1 \pm 2$ \\
\hline 4-Hydroxycarbazole (12) & - & $1 \pm 3$ & - & $-3 \pm 1$ \\
\hline 2-Hydroxycarbazole (13) & $190 \pm 18$ & $39 \pm 1$ & - & $12 \pm 6$ \\
\hline Harmalol (14) & - & $16 \pm 3$ & $95 \pm 14$ & $52 \pm 3$ \\
\hline Harmaline (15) & - & $-2 \pm 5$ & - & $0 \pm 2$ \\
\hline 3,4-Dihydro-7-hydroxy-2(1H)-quinolinone (16) & - & $-2 \pm 3$ & - & $-4 \pm 5$ \\
\hline Kojic acid (17) & $342 \pm 35$ & $31 \pm 10$ & $48 \pm 3$ & $68 \pm 1$ \\
\hline
\end{tabular}

a Values are means $\pm \mathrm{sd}$ (standard deviation), $n=3-4$.

b Calculated by subtracting the relative activity (\% of control) from 100 . A negative value means that the enzyme reaction is accelerated by the compound.

- , too large to be determined. 


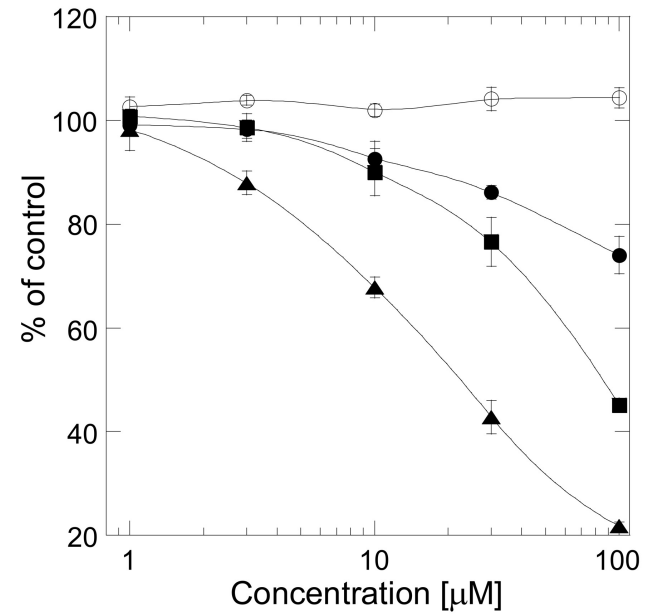

Fig. 2. Dose-dependency of the HMV-II tyrosinase inhibition by hydroxyindoles 4 (•), 5 (४), and 6 (•). 6-Hydroxyquinoline $(\mathbf{8}, \circ)$ was inactive. Values (\% of control) are the means \pm sd $(n=3)$ in a representative experiment that was repeated with comparable results.

of $100 \mu \mathrm{M} \mathbf{2}, \mathbf{6}$, and $\mathbf{8}$, respectively]. Similar phenomena with the mushroom enzyme have been reported for serotonin derivatives (Yamazaki et al., 2009).

The present result shows that hydroxyindoles are a unique class of mammalian tyrosinase inhibitors and that the position of the hydroxy group in the indole ring strongly affects the inhibitory activity. The best position is 6 , not 5 found previously with serotonin derivatives (Yamazaki et al., 2009). The 7-hydroxy group is also superior to the 5-hydroxy group for tyrosinase inhibition. On the other hand, 4- and 2-hydroxy groups are substantially ineffective on the inhibitory activity. A similar relationship is seen from the comparison of 2- (13) and 4-hydroxycarbazoles (12). The activity of an indole alkaloid, harmalol (14), and the inactivity of its $O$-methyl derivative, harmaline (15), also suggest the effectiveness of the 6 -hydroxy group (7-OH in $\beta$-carboline ring) in inhibiting tyrosinase. The inhibitory function of these hydroxy groups needs the indole structure, since the present analogues, 5-hydroxyindan (7), 6- (8) and 8-hydroxyquinoline (9), 4- (10) and 3-aminophenol (11), and 3,4-dihydro-7-hydroxy2(1H)-quinolinone $(\mathbf{1 6})$, are all weak or inactive inhibitors for the melanoma tyrosinase. The weak activity of $\mathbf{7}$ as compared to 5- (4) and 6-hydroxyindole (5) implies an essential role of the nitrogen atom that is located at the $\gamma$ or $\delta$ position to the phenolic hydroxy group. However, the contrasts of activities for $\mathbf{4} v s . \mathbf{8}$ and 10, 5 vs. 11 and 16, and $\mathbf{6} v s$. 9 suggest that the spatial arrangement of a phenolic hydroxy group and a nitrogen atom (or an NH group) is not a critical determinant for inhibition of tyrosinase. The electron density of the NH group and/or the whole structure of the ring should also be important for the inhibitory activity.

The inhibition mechanism of $\mathbf{4 , 5}$, and $\mathbf{6}$ for HMV-II tyrosinase was kinetically studied. The Lineweaver-Burk plots obtained with different concentrations of each compound cross on or very near the $Y$-axis (Fig. 3), indicating that these are
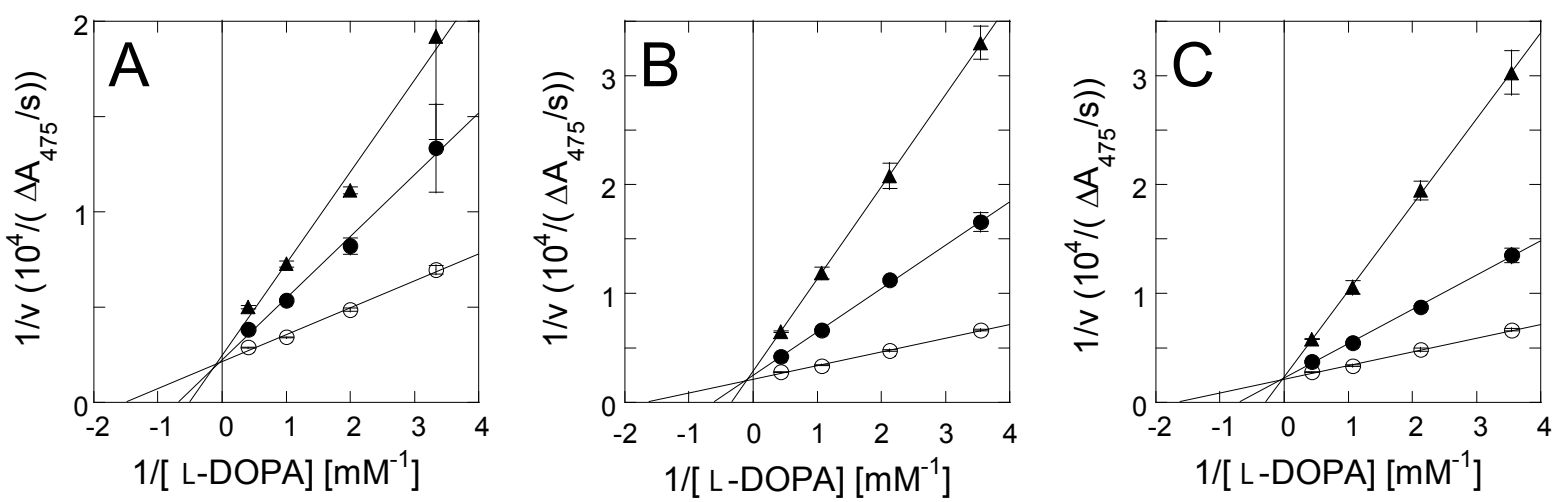

Fig. 3. HMV-II tyrosinase reaction kinetics in the presence of hydroxyindoles. (A) Lineweaver-Burk (L-B) plots for $4[0(\circ), 100(\bullet)$, and $300 \mu \mathrm{M}(\mathbf{\Delta})]$. (B) L-B plots for 5 [0 (०), $10(\bullet)$, and $30 \mu \mathrm{M}(\mathbf{\Delta})]$. (C) L-B plots for $\mathbf{6}[0$ (०), $30(\bullet)$, and $100 \mu \mathrm{M}(\Delta)]$. Every point is the mean $\pm \mathrm{sd}(n=3$ or 4$)$ and plot lines are drawn by least square linear regression. 
competitive inhibitors $\left(K_{\mathrm{i}}=160,6\right.$, and $18 \mu \mathrm{M}$ for $\mathbf{4 , 5}$, and $\mathbf{6}$, respectively) in the L-DOPA oxidation by HMV-II tyrosinase. Tyrosinase contains one binuclear copper complex in the catalytic centre and the catechol hydroxy groups of the substrate are considered to be bound to the copper atoms in the enzymatic oxidation process (Chang, 2009; Muñoz-Muñoz et al., 2008). Phenolic hydroxy groups are generally capable of coordinating copper atoms so that they inhibit the enzyme in competition with the catechol substrate. The competitive inhibition by the hydroxyindoles may arise in such a way. However, the present phenolics other than the hydroxyindole compounds were inactive or only weak active in inhibiting HMV-II tyrosinase. Even 8-hydroxyquinoline (9) that is a strong copper-chelating ligand (Perrin, 1979) was almost inactive. Therefore, the copper-binding ability of a phenol group alone is not enough to perform the tyrosinase inhibition. This primary binding to one copper atom should be strengthened by additional interactions between the indole ring (e.g., the $\mathrm{NH}$ group and $\pi$ electrons of the ring) and another copper atom or the amino acid residues of the enzyme's active site to accomplish the inhibition. Since 5,6-dihydroxyindole is an intermediate of melanin biosynthesis and is also a substrate of tyrosinase (Chang, 2009), it is likely that 6-hydroxyindole (5) has the sites that crucially interact with the enzyme. 6- (5) and 7-hydroxyindole (6) are reported to be useful in controlling hair colouration (i.e., as melanin precursors) (Cotteret and Audousset, 1993), but little is known about their tyrosinase inhibitory activity. The present study reveals that $\mathbf{5}$ is considerably a stronger inhibitor than the other hydroxyindoles $\mathbf{2}, \mathbf{3}, \mathbf{4}$, and 6 for HMV-II tyrosinase, showing that the position of the hydroxy group in a specific heterocyclic ring such as in indole is possibly optimized to produce more potent inhibitors against human tyrosinase.

Bouchard B., Fuller B., Vijayasaradhi S., and Houghton A. N. (1989), Induction of pigmentation in mouse fibroblasts by expression of human tyrosinase cDNA. J. Exp. Med. 169, 2029-2042.

Cabanes J., García-Carmona F., García-Cánovas F., Iborra J. L., and Lozano J. A. (1984), Kinetic study on the slow inhibition of epidermis tyrosinase by $m$-coumaric acid. Biochim. Biophys. Acta 790, 101-107.

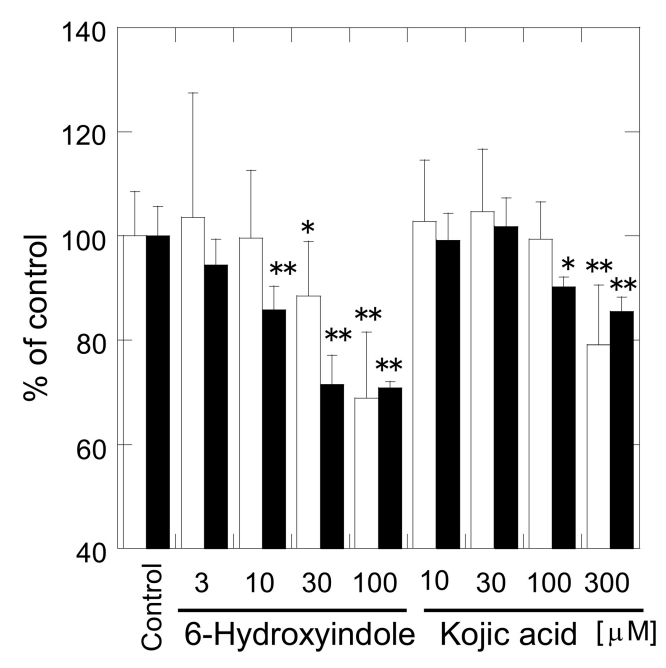

Fig. 4. Antimelanogenic activity and cytotoxicity of 6-hydroxyindole (5) and kojic acid (17) in a HMV-II cell culture. Values ( $\%$ of control) are the means \pm sd ( $n=3-4$ for melanin and $n=8-10$ for cell viability) in a representative experiment that was repeated with comparable results. Closed bars, melanin content; open bars, cell viability. $* *$ and $*$ represent $p<0.01$ and $p<$ 0.05 versus control by Student's $t$-test, respectively.

The antimelanogenic activity of $\mathbf{5}$ was evaluated in a culture of HMV-II melanoma cells. The melanin formation was suppressed by $14 \%$ with 5 at a concentration of $10 \mu \mathrm{M}$, where the cell viability was not significantly different from the control (Fig. 4). On the other hand, $10 \mu \mathrm{m}$ kojic acid (17) showed no antimelanogenic activity in the same experimental conditions as above. The melanin suppression by 5 increased up to $25-30 \%$ at 30 or $100 \mu \mathrm{M}$, while the cell viability also significantly decreased. Thus, 6-hydroxyindole (5) is a potentially useful pharmacophore of antimelanogenic agents, but the solution of the problem of cytotoxicity, for example, by chemical modifications has to be studied in the future.

Chang T.-S. (2009), An updated review of tyrosinase inhibitors. Int. J. Mol. Sci. 10, 2440-2475.

Cho S. J., Roh J. S., Sun W. S., Kim S. H., and Park K. D. (2006), $N$-Benzylbenzamides: a new class of potent tyrosinase inhibitors. Bioorg. Med. Chem. Lett. 16, 2682-2684.

Cotteret J. and Audousset M. P. (1993), Oxidative hair dye comprising 2 -substituted $p$-aminophenols com- 
bined with 6- or 7-hydroxyindole. PCT Int. Appl. WO 9320794; Chem. Abstr. 120, 14639.

Khan M. T. H. (2007), Molecular design of tyrosinase inhibitors: a critical review of promising novel inhibitors from synthetic origins. Pure Appl. Chem. 79, 2277-2295.

Kim Y. M., Yun J., Lee C.-K., Lee H., Min K. R., and Kim Y. (2002), Oxyresveratrol and hydroxystilbene compounds. Inhibitory effect on tyrosinase and mechanism of action. J. Biol. Chem. 277, 16340-16344.

Kubo I., Kinst-Hori I., Chaudhuri S. K., Kubo Y., Sánchez Y., and Ogura T. (2000), Flavonols from Heterotheca inuloides: tyrosinase inhibitory activity and structural criteria. Bioorg. Med. Chem. 8, 1749-1755.

Kubo I., Chen Q. X., Nihei K.-I., Calderón J. S., and Céspedes C. L. (2003), Tyrosinase inhibition kinetics of anisic acid. Z. Naturforsch. 58c, 713-718.

Liu S.-H., Pan I.-H., and Chu I.-M. (2007), Inhibitory effect of $p$-hydroxybenzyl alcohol on tyrosinase activity and melanogenesis. Biol. Pharm. Bull. 30, $1135-1139$.

Muñoz-Muñoz J. L., García-Molina F., García-Ruiz P. A., Molina-Alarcón M., Tudela J., García-Cánovas F., and Rodríguez-López J. N. (2008), Phenolic substrates and suicide inactivation of tyrosinase: kinetics and mechanism. Biochem. J. 416, 431-440.
Nishida J., Gao H., and Kawabata J. (2007), Synthesis and evaluation of 2',4',6'-trihydroxychalcones as a new class of tyrosinase inhibitors. Bioorg. Med. Chem. 15, 2396-2402.

Okombi S., Rival D., Bonnet S., Mariotte A.-M., Perrier E., and Boumendjel A. (2006), Analogues of $N$ hydroxycinnamoylphenalkylamides as inhibitors of human melanocyte-tyrosinase. Bioorg. Med. Chem. Lett. 16, 2252-2255.

Perrin D. D. (1979), IUPAC Chemical Data Series No. 22, Stability Constants of Metal-Ion Complexes Part B: Organic Ligands. Pergamon Press, Oxford, pp. 649-651.

Pomerantz S. H. (1963), Separation, purification, and properties of two tyrosinases from hamster melanoma. J. Biol. Chem. 238, 2351-2357.

Seiji M. (1961), Isolation and properties of tyrosinases from melanomas of Swiss white and C-57 black mice. Federation Proc. 20, 6.

Tamura S., Nitoda T., and Kubo I. (2007), Effects of salicylic acid on mushroom tyrosinase and B16 melanoma cells. Z. Naturforsch. 62c, 227-233.

Yamazaki Y., Kawano Y., Yamanaka A., and Maruyama S. (2009), $N$-[(Dihydroxyphenyl)acyl]serotonins as potent inhibitors of tyrosinase from mouse and human melanoma cells. Bioorg. Med. Chem. Lett. 19, 4178-4182. 